\title{
Pericardiectomia parcial toracoscópica em um cão
}

\author{
Thoracoscopic partial pericardectomy in a dog \\ Fernanda Voll Costa Ventura, Rafael Stedile, Carlos Afonso de Castro Beck, \\ Simone Tostes de Oliveira, Elisa Barp Neuwald, Vivian Cristina Schwantes, \\ Fabiana Schiochet, Simone Scherer \& Ruben Lundgren Cavalcanti
}

\begin{abstract}
RESUMO
A efusão pericárdica em cães frequentemente é idiopática ou secundária à neoplasia. O acúmulo de líquido causa aumento da pressão intrapericárdica e alterações hemodinâmicas. Este trabalho relata o tratamento cirúrgico através de pericardiectomia parcial toracoscópica em um cão com efusão pericárdica recidivante. Acredita-se que esta seja a primeira descrição da utilização desta técnica, em caso clínico, no Brasil. Foi atendido, no Hospital de Clínicas Veterinárias da UFRGS, um cão Akita, macho, de 11 anos, com sinais clínicos compatíveis com efusão pericárdica, confirmada através de exames complementares (radiografia torácica, eletrocardiografia e ecografia). Após duas pericardiocenteses com recidiva do acúmulo de líquido, o paciente foi submetido à videotoracoscopia intercostal para realização de pericardiectomia parcial. Após 76 dias da cirurgia, o animal veio a óbito, contudo não decorrente de efusão pericárdica. No presente caso, a técnica minimamente invasiva se mostrou efetiva, sendo considerada rápida, de fácil realização e sem complicações pós-operatórias significativas.
\end{abstract}

Descritores: efusão pericárdica, pericardiocentese, toracoscopia, videocirurgia, cirurgia torácica, tórax.

\begin{abstract}
The pericardial effusion in dogs is frequently idiopathic or secondary to neoplasia. Fluid accumulation causes increased intrapericardial pressure and hemodynamic alterations. A surgical treatment through thoracoscopic partial pericardectomy in a dog with recurrent pericardial effusion is described. To the authors' knowledge, this technique has not been reported for clinical use in Brazil. An 11-year-old male Akita was presented at the Hospital de Clínicas Veterinárias of UFRGS with clinical signs compatible to pericardial effusion, which was confirmed through complementary exams (thoracic radiography, electrocardiography and echocardiography). After two pericardiocentesis with recurrence of fluid accumulation, the patient was submitted to intercostal thoracoscopy for performing partial pericardectomy. The patient died 76 days post-surgery; however it was not due to pericardial effusion. The procedure was technically successful. In the present report, the minimally invasive surgery was easy and rapid to perform, and with no significant postoperative complications.
\end{abstract}

Keywords: pericardial effusion, pericardiocentesis, thoracoscopy, endosurgery, thoracic surgery, thorax. 


\section{INTRODUÇÃO}

A efusão pericárdica é definida como um acúmulo anormal de fluido dentro do saco pericárdico $[5,9,15]$. Sinais clínicos inespecíficos como abafamento dos sons cardíacos, pulso fraco, distensão abdominal, perda de peso, dificuldade respiratória, anorexia ou inapetência e letargia são associados às efusões, independentemente da causa do derrame [16,17].

$\mathrm{O}$ tratamento da efusão requer pericardiocentese, e quando mais que duas drenagens são necessárias, ou se uma neoplasia está presente, a pericardiectomia é recomendada [7,12]. A pericardiectomia pode ser realizada por acessos convencionais, tais como esternotomia mediana e toracotomia lateral direita ou por cirurgia videoassistida de acesso mínimo (toracoscopia) [14]. A toracoscopia apresenta vantagens em relação aos acessos convencionais, com redução da dor pós-operatória, menor tempo de recuperação, melhor aspecto cosmético e diminuição das complicações das feridas cirúrgicas [18]. O objetivo deste trabalho é relatar a pericardiectomia parcial por toracoscopia em um cão com efusão pericárdica.

\section{RELATO DE CASO}

Um canino macho, da raça Akita, de 11 anos, pesando $35 \mathrm{~kg}$, foi atendido com histórico de apatia, inapetência, perda de peso e dispnéia. Ao exame clínico, percebeu-se distensão abdominal, mucosas pálidas, tempo de perfusão capilar aumentado, distensão venosa jugular, pulso hipocinético e sons cardíacos abafados. Foram realizados exames laboratoriais, eletrocardiograma, radiografia torácica e ecografia.

O hemograma revelou anemia normocítica normocrômica arregenerativa; trombocitopenia, hipoproteinemia; leucocitose devido à neutrofilia com desvio à direita, presença de neutrófilos tóxicos e linfopenia. A bioquímica sanguínea incluiu dosagens de alanina aminotransferase, fosfatase alcalina, uréia e creatinina, que se encontravam normais. O eletrocardiograma revelou a presença de bloqueio atrioventricular de $1^{\circ}$ grau, baixa amplitude dos complexos QRS e a presença de alternância elétrica (Figura 1). Evidenciou-se aumento global da silhueta cardíaca no exame radiográfico (Figura 2). A ecografia confirmou o derrame pericárdico e a ascite. A pericardiocentese e a abdominocentese permitiram retirar, respectivamente, 200 e $3000 \mathrm{~mL}$ de um líquido de coloração avermelhada. Após a drenagem, percebeu-se melhora hemodinâmica. O cão retornou para casa com prescrição de diuréticos e inibidor da enzima conver-sora de angiotensina. A análise laboratorial do líquido pericárdico apresentou neutrófilos não degenerados (44\%), linfócitos (26\%), monócitos (5\%) macrófagos (8\%), 23\% de hematócrito, células mesoteliais reativas com nucléolos evidentes, ausência de plaquetas e pH 7,5.

Após duas recidivas de efusão pericárdica, tratadas com pericardiocentese, em um período de três meses, o cão foi encaminhado para pericardiectomia parcial por toracoscopia. A meperidina (3mg. $\left.\mathrm{kg}^{-1}\right)$ foi utilizada como medicação pré-anestésica, seguida por indução anestésica com midazolam $\left(0,5 \mathrm{mg} \cdot \mathrm{kg}^{-1}\right)$ e etomidato $\left(1,5 \mathrm{mg} \cdot \mathrm{kg}^{-1}, \mathrm{IV}\right)$, na mesma seringa. A manu-tenção da anestesia foi feita com isofluorano vaporizado em circuito semiaberto com oxigênio. A analgesia transoperatória foi obtida com infusão intravenosa de citrato de fentanila ( $\left.5 \mu \mathrm{g} . \mathrm{kg}^{-1}\right)$, em bolus. O bloqueio dos nervos intercostais foi feito por infusão de lidocaína $2 \%$. Ampicilina sódica $\left(22 \mathrm{mg} \cdot \mathrm{kg}^{1}\right.$, IV) foi utilizada como antibiótico profilático.

O animal foi posicionado em decúbito lateral esquerdo e realizada tricotomia ampla em parede torácica direita e antissepsia. Para acesso à cavidade

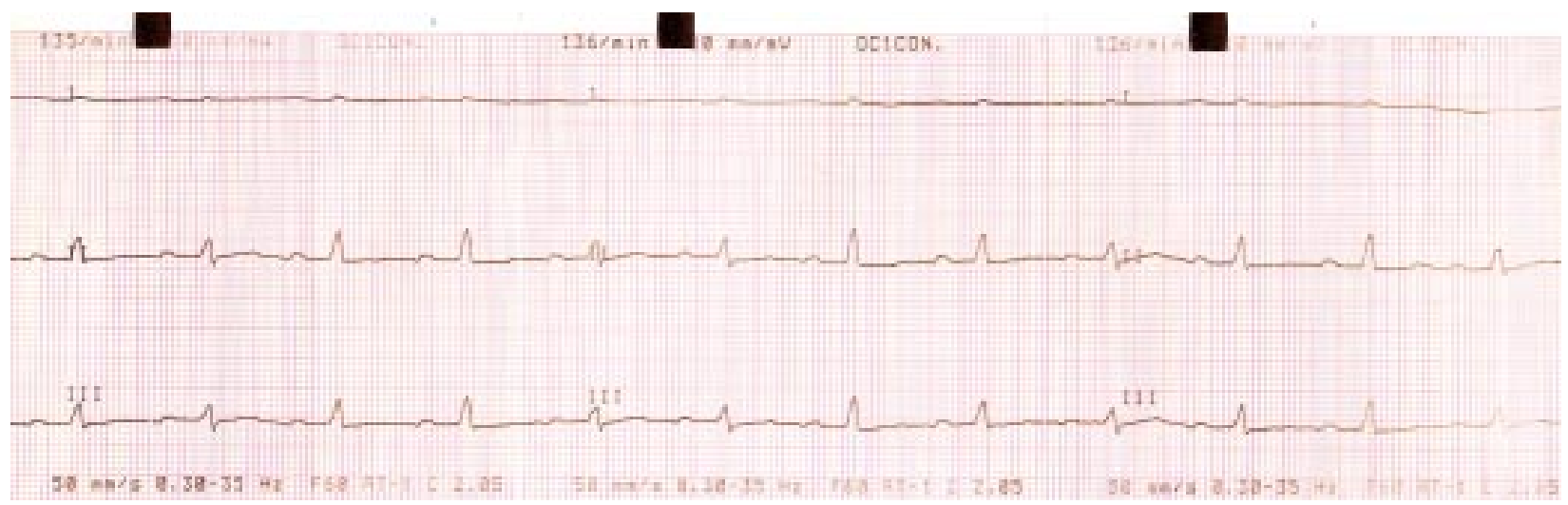

Figura 1. Eletrocardiograma de cão com efusão pericárdica. O traçado mostra a presença de bloqueio atrioventricular de $1^{\circ}$ grau, baixa amplitude dos complexos QRS e alternância elétrica. 


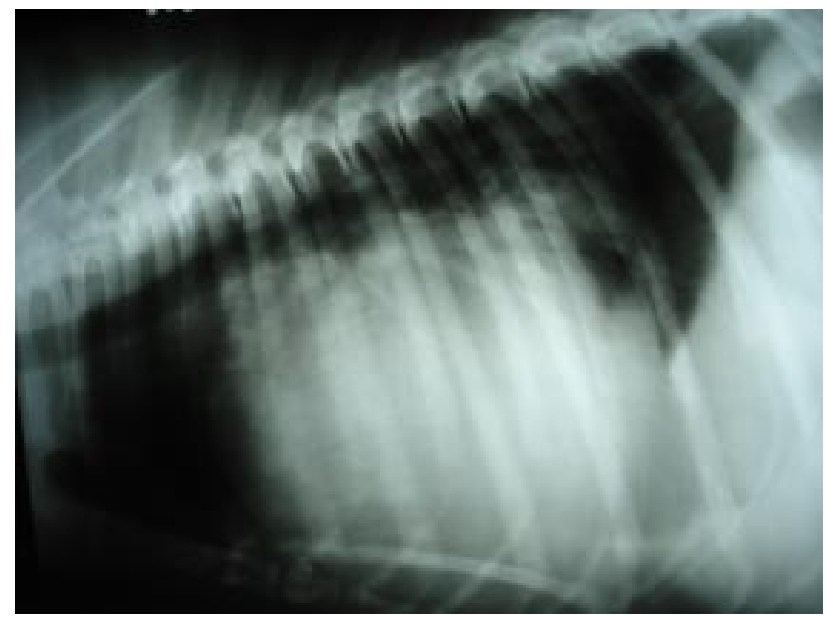

Figura 2. Radiografia torácica de cão, mostrando silhueta cardíaca globosa, sugestivo de efusão pericárdica.

torácica, efetuou-se incisão de pele e tecido subcutâneo de aproximadamente $1 \mathrm{~cm}$, entre o terço médio e dorsal do sexto espaço intercostal direito. Fez-se divulsão romba da musculatura até a perfuração da pleura. Introduziu-se um trocarte de $10 \mathrm{~mm}$ curto com rosca para passagem da óptica de $10 \mathrm{~mm}$. Instituiu-se pressão de $3 \mathrm{mmHg}$ com dióxido de carbono. Inspecionou-se a cavidade com identificação da distensão pericárdica. A colocação de dois trocartes curtos com rosca de $5 \mathrm{~mm}$ e $10 \mathrm{~mm}$, que serviram de portais de trabalho, foi realizada sob visualização direta no terço médio do tórax no quinto e oitavo espaços intercostais direito, respectivamente. Com o auxilio da pinça de apreensão, elevou-se o pericárdio e seccionou-se o mesmo, criando uma a janela de aproximadamente $4 \times 4 \mathrm{~cm}$. Removeu-se o fragmento do pericárdio através do redutor de $5 \mathrm{~mm}$ (Figura 3). O pericárdio encontrava-se espessado. Aspirou-se o líquido livre na cavidade torácica oriundo do derrame pericárdico. Os trocartes foram removidos e a musculatura intercostal aproximada com sutura padrão Sultan e redução do espaço morto subcutâneo com ácido poliglicólico 2-0. A dermorrafia foi efetuada com mononáilon 3-0 em sutura padrão isolado simples. A pressão intratorácica foi restaurada utilizando sistema de drenagem com torneira de três vias. O tempo cirúrgico total foi de 37 minutos, sendo que o tempo para pericardiectomia foi de sete minutos. O animal apresentou recuperação anestésica tranquila e sem maiores complicações. No período pós-operatório, utilizou-se cetoprofeno $\left(2 \mathrm{mg} \mathrm{kg}^{-1}\right)$ via intramuscular (IM), a cada $24 \mathrm{~h}$, por três dias e cloridrato de tramadol $\left(2 \mathrm{mg} \cdot \mathrm{kg}^{-1}\right), \mathrm{IM}$, a cada 8 horas por 24 horas. Os pontos foram removidos em oito dias.

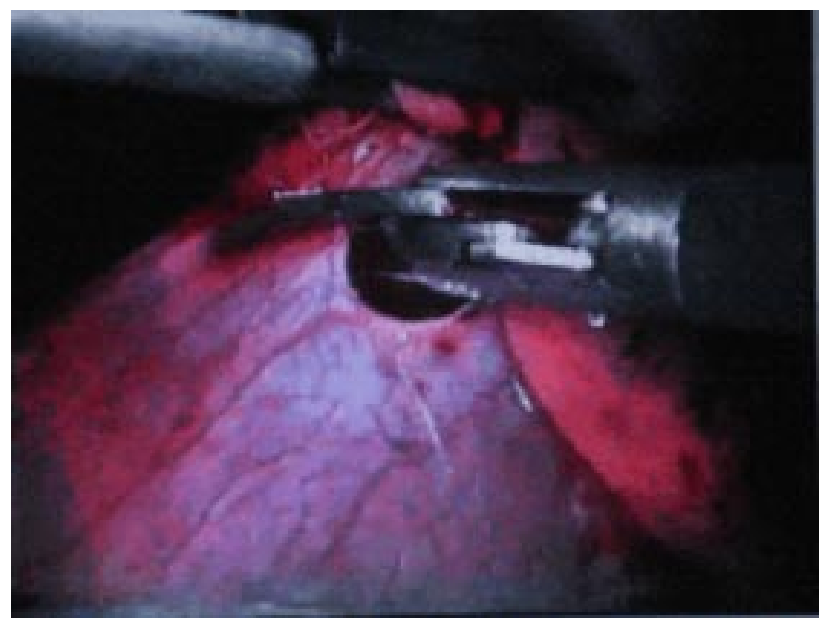

Figura 3. Retirada de fragmento de pericárdio do cão, através de acesso toracoscópico.

A avaliação histopatológica do fragmento pericárdico foi compatível com inflamação crônica com fibrose. O cão retornou com efusão pleural, porém sem efusão pericárdica, 76 dias após a cirurgia. Apesar de ter sido submetido à drenagem, não apresentou melhora clínica e veio a óbito em 24 horas. Os proprietários não autorizaram a necropsia.

\section{DISCUSSÃO}

A suspeita de efusão pericárdica no presente relato baseou-se nos achados clínicos compatíveis com o descrito pela bibliografia, principalmente em relação à hemodinâmica. Estes sinais estão relacionados ao aumento da pressão intrapericárdica, à medida que aumenta o acúmulo de líquido. As alterações progressivas na função diastólica decorrentes deste acúmulo levam à redução no débito cardíaco e à elevação da pressão venosa sistêmica, culminando na manifestação de sinais de insuficiência cardíaca [7,12].

As neoplasias constituem as causas mais comuns de efusão pericárdica em cães, seguidas da efusão pericárdica idiopática, esta freqüentemente vista em cães machos idosos de raças grandes [7]. Outras causas incluem ruptura atrial, trauma, hipoproteinemia, insuficiência cardíaca congestiva, causas congênitas, pericardite infecciosa e uremia $[3,5-7,9,10,15]$. $\mathrm{O}$ exame radiográfico e a eletrocardiografia também foram sugestivos de efusão pericárdica. Apesar da baixa voltagem do complexo QRS ser uma alteração quase sempre presente no eletrocardiograma de animais portadores de efusão pericárdica, é considerada um indicador fraco da presença de derrame pericárdico quando avaliada isoladamente. A alternância elétrica, observada no presente caso, é variação alternada no 
tamanho do complexo QRS, decorrente da movimentação do coração dentro do derrame pericárdico. Esta alteração é encontrada em $50 \%$ dos pacientes com efusão pericárdica, sendo geralmente mais evidente na presença de grandes volumes intrapericárdicos [12,19]. Como citado na bibliografia, a ecocardiografia serviu tanto para confirmação de efusão pericárdica, como para a investigação de alterações compatíveis com neoplasias cardíacas ou pericárdicas, as quais não foram observadas [7,19]. Contudo, a não identificação de uma massa não exclui a sua presença, visto que a ecocardiografia não é sensível em detectar neoplasias cardíacas $[4,7,10]$.

A diferenciação entre efusão de etiologia neoplásica ou não neoplásica in vivo constitui um desafio [10,11], especialmente no que diz respeito à distinção entre efusão idiopática benigna e mesotelioma [8]. A análise e a citologia do fluido pericárdico não constituem método totalmente confiável para fins de diagnóstico definitivo $[4,15]$. A análise do $\mathrm{pH}$ do fluido pericárdico na diferenciação da etiologia considera valores de $\mathrm{pH}$ acima de 7,3 como sendo associados à ocorrência de neoplasias e pH abaixo de 7,2 como relacionado com efusões não neoplásicas [10].

A pericardiocentese é o tratamento de escolha em casos de efusões pericárdicas, independentemente da etiologia, no entanto, a recidiva de derrame justifica a pericardiectomia a fim de eliminar o risco de morrer por tamponamento [3,5]. O tratamento conservador utilizado neste cão está de acordo com o recomendado pela bibliografia, assim como a opção cirúrgica após a segunda recidiva.

$\mathrm{O}$ acesso toracoscópico intercostal permitiu adequada visualização do hemitórax direito e do sitio operatório, com magnificação das estruturas.
A distribuição dos portais seguiu as utilizadas por Beck e colaboradores em pericardiectomias experimentais [2]. A toracoscopia paraxifóide transdiafragmática também está descrita para este procedimento [13]. Como não parece haver diferença quanto à eficiência da técnica quando compara-se a pericardiectomia total com a parcial, sendo a pericardiectomia parcial relativamente mais simples e com menor risco [3], optou-se pela última, no presente relato. Semelhante ao observado em redução de hérnias diafragmáticas por toracoscopia, também não houve necessidade da realização da ventilação seletiva pulmonar para realização da pericardiectomia parcial, no presente caso [1]. A recuperação pós-operatória do paciente, rápida, sem complicações ou sinais de dor corrobora com o citado na literatura para esta técnica cirúrgica [14,18]. Acredita-se que esta seja a primeira descrição da utilização desta técnica em caso clínico no Brasil.

Um estudo mostrou que a complicação mais comum após pericardiectomia é a efusão pleural e que sua ocorrência num período maior que 30 dias após o procedimento estava associada ao mau prognóstico e na maioria dos casos, à etiologia neoplásica [10]. Apesar dos aspectos clínicos e exames complementares sugerirem efusão idiopática benigna, o curto tempo de sobrevivência após pericardiectomia, os dados da análise do fluido pericárdico e a não realização de necropsia não permitem excluir o diagnóstico de neoplasia.

\section{CONCLUSÃO}

No presente caso, a pericardiectomia parcial toracoscópica se mostrou efetiva no tratamento da efusão pericárdica recidivante em um cão, sendo considerada rápida, de fácil realização e sem complicações pós-operatórias significativas.

\section{REFERÊNCIAS}

1 Beck C.A.C., Pippi N.L., Brun M.V, Contesini E.A., Cunha A.F., Stedile R., Bonfada A.T., Silva Filho A.P.F., Gomes K. \& Colomé L.M. 2004. Toracoscopia em hérnias diafragmáticas: estudo experimental em cães. Ciência Rural. 34 : $1857-$ 1863.

2 Beck C.A.C., Stedile R., Schiochet F., Freire C.D., Santos Junior E.B., Sia D.B., Pellizzari M.L. \& Silva Filho A.P.F. 2004. Pericardiectomia parcial pelo acesso toracoscópico em cão. In: Anais do Congresso Brasileiro de Videocirurgia (Porto Alegre, Brasil). p.45. [Fonte: <http://www.ufrgs.br/medanimal/anais.pdf>].

3 Berg R.J., Wingfield W.E. \& Hoopes P.J. 1984. Idiopathic hemorrhagic pericardial effusion in eight dogs. Journal of American Veterinary Medical Association. 185: 988-992.

4 Bouvy B.M. \& Bjorling D.E. 1991. Pericardial effusion in dogs and cats, part II: diagnostic approach and treatment. The Compendium on Continuing Education for the Practicing Veterinarian. 13: 633-641.

5 Dunning D., Monnet E., Orton C. \& Salman M.D. 1998. Analysis of prognostic indicators for dogs with pericardial effusion: 46 cases (1985-1996). Journal of the American Veterinary Medical Association. 212: 1276-1280. 
6 Edwards N.J. 1996. The diagnostic value of pericardial fluid pH determination. Journal of the American Animal Hospital Association. 32: 63-67.

7 Jinks J. 2001. Pericardial effusion in a mixed breed dog. Canadian Veterinary Journal. 42:877-879.

8 Johnson M.S., Martin M., Binns S.E. \& Day M.J. 2004. A retrospective study of clinical findings, treatment and outcome in 143 dogs with pericardial effusion. Journal of Small Animal Practice. 45: 546-552.

9 Kerstetter K.K., Krahwinkel D.J., Millis D.L. \& Hahn K. 1997. Pericardectomy in dogs: 22 cases (1978-1994). Journal of the American Veterinary Medical Association. 211: 736-739.

10 Kirsch J.A., Dhupa S. \& Cornell K.K. 2000. Pericardial effusion associated with metastatic disease from an unknown primary tumor in a dog. Journal of American Animal Hospital Association. 36: 121-124.

11 Machilda N., Tanaka R., Takemura N., Fujii Y., Ueno A. \& Mitsumori K. 2004. Pericardial mesothelioma in dogs with IHPE. Journal of Comparative Pathology. 131: 166-175.

12 Miller M.W. \& Sisson D.D. 2004. Distúrbios Pericárdicos. In: Ettinger S.J. \& Feldman E.C. (Eds). Tratado de Medicina Interna Veterinária: Doenças do Cão e do Gato. 5.ed. Rio de Janeiro: Guanabara Koogan, pp.978-991.

13 Potter L. 1998. Video-assisted thoracic surgery. In: Freeman L.J. (Ed). Veterinary Endosurgery. Saint Louis: Mosby, pp.169-187.

14 Shaw S.P. \& Rush J.E. 2007. Canine pericardial effusion: diagnostic, treatment, and prognosis. The Compendium on Continuing Education for the Practicing Veterinarian. 29: 405-411.

15 Sisson D., Thomas W.P., Ruehl W.W. \& Zinkl J.G. 1984. Diagnostic value of pericardial fluid analysis in the dog. Journal of the American Veterinary Medical Association. 184: 51-55.

16 Stepien R.L., Whitley N.T. \& Dubielzig R.R. 2000. Idiopathic or mesothelioma-related pericardial effusion: clinical findings and survival in 17 dogs studied retrospectively. Journal of Small Animal Practice. 41: 342-347.

17 Vicari E.D., Brown D.C., Holt D.E. \& Brockman D.J. 2001. Survival times of and prognostic indicators for dogs with heart base masses: 25 cases (1986-1999). Journal of the American Veterinary Medical Association. 219: 485-487.

18 Walsh P.J., Remedios A.M., Ferguson J.F., Walker D.D., Cantwell S. \& Duke T. 1999. Thoracoscopic versus open partial pericardectomy in dogs: comparison of postoperative pain and morbidity. Veterinary Surgery. 28: 472-479.

19 Ware W.E. 2006. Distúrbios pericárdicos e tumores cardíacos. In: Nelson R.W. \& Couto C.G. (Eds). Medicina Interna de Pequenos Animais. 3.ed. Rio de Janeiro: Elsevier, pp.181-192. 